\title{
Cotoneaster manna oral drop for the management of neonatal hyperbilirubinemia; a randomized, double- blinded and placebo controlled clinical trial
}

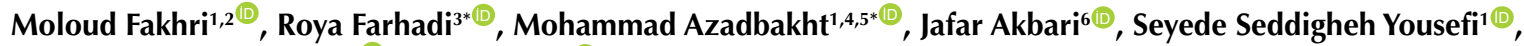 \\ Nouraldin Mousavinasab $^{7 \oplus}$, Ali Davoodi ${ }^{(\mathbb{B}}$ \\ ${ }^{1}$ Traditional and Complementary Medicine Research Center, Addiction Institute, Mazandaran University of Medical Sciences, Sari, Iran \\ ${ }^{2}$ Faculty of Medicine, Mazandaran University of Medical Sciences, Sari, Iran \\ ${ }^{3}$ Department of Pediatrics, Faculty of Medicine, Bouali Hospital, Mazandaran University of Medical Sciences, Sari, Iran \\ ${ }^{4}$ Department of Pharmacognosy, Faculty of Pharmacy, Mazandaran University of Medical Sciences, Sari, Iran \\ ${ }^{5}$ The Health of Plant and Livestock Products Research Center, Mazandaran University of Medical Sciences, Sari, Iran \\ ${ }^{6}$ Department of Pharmaceutics, Faculty of Pharmacy, Mazandaran University of Medical Sciences, Sari, Iran \\ ${ }^{7}$ Department of Biostatistics, Faculty of Health, Mazandaran University of Medical Sciences, Sari, Iran
}

\author{
*Correspondence to \\ Mohammad Azadbakht, Email: \\ Azadbakht110@gmail.com \\ mazadbakht@mazums.ac.ir, \\ and Roya Farhadi, \\ Email: \\ dr_royafarhadi@yahoo.com, \\ rfarhadi@mazums.ac.ir
}

Received 6 Aug. 2020 Accepted 3 Nov. 2020 Published online 28 Nov. 2020

Keywords: Cotoneaster manna Neonatal jaundice, Bilirubin, Medicinal plants

\section{Abstract}

Introduction: Neonatal hyperbilirubinemia is a common physiologic condition, which is occurred in early days after birth with an estimated incidence of about $50 \%$ in term neonates.

Objectives: The aim of this study was to evaluate the effect of Cotoneaster nummularioides manna on reducing neonatal total serum bilirubin (TSB) levels, and shortening hospitalization period.

Patients and Methods: One hundred breast-fed term neonates with hyperbilirubinemia were randomly assigned to the intervention group or control group. The Newborns of the intervention group were received Cotoneaster manna drop in the dosage of $1 \mathrm{~g} / \mathrm{d}$ divided into three doses every 8 hours for at least 3 days and the control group received placebo drops. Total serum bilirubin levels were measured at baseline, at 12, 24 hours and then daily through hospitalization days (day 9). Secondary outcome (discharge from hospital, TSB $<10 \mathrm{mg} / \mathrm{dL}$ ) was also documented with risk ratio calculation.

Results: TSB levels were declined in both groups; however, this was more prominent in the manna group than the placebo group. The greatest difference of mean TSB levels between the intervention and control groups was seen on days two $(10.01$ versus $13.04 \mathrm{mg} / \mathrm{dL}$; mean difference $[95 \% \mathrm{Cl}]:-3.03[-3.60$ to -2.46$]$; $P<0.001)$. On day four, significantly more newborns in the intervention group versus the control group were discharged $(70 \%$ versus $6.1 \%$; risk ratio $=11.43,95 \% \mathrm{Cl}=3.76,34.74)$. No major side effects were recorded in both groups.

Conclusion: Cotoneaster nummularioides manna drop combined with phototherapy was superior to the control group in reducing TSB levels and reaching this observation more rapidly and shortening hospitalization period with no major side effects.

Trial Registration: The trial protocol was approved by the Iranian Registry of Clinical trials (identifier: IRCT20160531028188N4; https://en.irct.ir/trial/27765, ethical code \#IR.MAZUMS.REC.1394.1316).

Citation: Fakhri M,
Farhadi R, Azadbakht
M, Akbari J, Yousefi
SS, Mousavinasab N,
Davoodi A. Cotoneaster
manna oral drop for the
management of neonatal
hyperbilirubinemia; a
randomized, double-
blinded and placebo
controlled clinical
trial. Immunopathol
Persa. 2022;8(1):e10.
DOI:10.34172/
ipp.2022.10.

\section{Introduction}

Neonatal hyperbilirubinemia is a common physiologic condition, which is occurred in early days after birth with an estimated incidence of about $50 \%$ in term neonates $(1,2)$. Neonatal jaundice usually occurs in the first week of life. The elevated serum bilirubin is usually benign in most neonates and is termed physiologic jaundice that responds well to phototherapy. On the other hand, pathological jaundice, in particular, severe hyperbilirubinemia (generally defined as total serum bilirubin [TSB] level bilirubin $>25 \mathrm{mg} / \mathrm{dL}$ ) is less common (8-9\%) (3), which may result in accumulation of unconjugated bilirubin in the infant's tissues such as brain tissue and cause bilirubin-induced neurologic dysfunction and kernicterus $(1,4)$. The phototherapy and exchange transfusion have been applied for mild and severe cases of hyperbilirubinemia in neonatal period, respectively. Besides the above-mentioned treatments, some newer interventions have been advocated as potential treatments for neonatal jaundice. For example, a recent review of 13 randomized clinical trials (5) showed that supplementation with probiotics is a safe and effective intervention to manage

Copyright $\odot 2022$ The Author(s); Published by Nickan Research Institute. This is an open-access article distributed under the terms of the Creative Commons Attribution License (http://creativecommons.org/licenses/by/4.0), which permits unrestricted use, distribution, and reproduction in any medium, provided the original work is properly cited. 


\section{Key point}

Total serum bilirubin levels were declined in both groups; however, this was more prominent in the manna group than placebo group. The greatest difference of mean TSB levels between the intervention and control groups was seen on day 2 . On day 4 , significantly more newborns in the intervention group versus control group were discharged. No major side effects were recorded in both groups. Cotoneaster nummularioides manna drop combined with phototherapy was superior to control group in reducing TSB levels and reaching this observation more rapidly and shortening hospitalization period with no major side effects.

pathological jaundice in infants. This observation was attributed to the effect of probiotics in enhancing the immune system of the neonate via preventing translocation of bacteria as well as regulation of gut bacterial colonies. Herbal medicines have also been investigated. In a recently published meta-analysis on Yinzhihuang oral liquid/ solution, which is a Chinese herbal preparation, alone or in combination with other conventional treatments TSB and duration of jaundice effectively decreased in newborns (6). Another medicinal herb that has been studied in neonatal jaundice is the genus Cotoneaster (Purgative manna) which belongs to the Rose family (Rosaceae) and includes about 50 species of shrubs and small trees. Cotoneaster is indigenous to temperate Eurasia and several species of this plant have been used as medicinal plants in areas like Iran, Turkey, Himalayas, Mongolia, and China $(7,8)$. This is called "Shir-e-Khesht" in Iran and its secondary exudate product called "Manna". This plant has been used in traditional medicine and local people for a long time in conditions such as fever, cough, abnormal menstruation, and neonatal jaundice $(7,8)$. Neonatal jaundice in Asia, especially in developing countries, is very important in this region. Persian medicine has specific guidelines for various types of herbal medicines, including infant formula, which can be cost-effective by adapting them to new science and research (9). As there is promising evidence regarding the efficacy of Cotoneaster manna in neonatal jaundice and paucity of sufficient scientific evidence with this regard, it is necessary to conduct further clinical trials to determine the efficiency and safety of Cotoneaster manna in neonatal jaundice.

\section{Objectives}

In this clinical trial, we intended to compare the therapeutic effect of Cotoneaster manna versus placebo in addition to phototherapy in the treatment of neonatal jaundice for the first time. The hypotheses of this trial were whether Cotoneaster nummularioides manna can result in more rapid improvement of neonatal jaundice and whether this treatment can shorten hospitalization duration. In our opinion, the results of this trial will highlight new insights regarding the role of this herbal medicine for management of a relatively common neonatal condition.

\section{Patients and Methods}

\section{Oral drop formulation and analysis}

The purgative manna (C. nummularioides Pojark) used in this study, was analyzed in our previous study and its chemical characterization found in the previous paper (10). The purgative manna (C. nummularioides Pojark) was used in this study. Manna was dissolved in deionized water, and its foreign matter was removed. The watersoluble content was concentrated, dried and used for drop preparation. Moreover, propyl paraben and methyl paraben were used as preservative. In addition, physicochemical and microbial controls and effect of preservatives were performed based on the USP XXIII procedure.

\section{Trial design}

This was a single-center double-blinded parallelrandomized clinical trial (RCT) with superiority who received phototherapy and Cotoneaster manna drop. The other group consisted of neonates with hyperbilirubinemia who received placebo drop with phototherapy.

\section{Participants}

The eligible subjects consisted of term neonates (gestational age of 38 to 42 weeks) admitted to "roaming in" (keeping with mother) the neonate department of Boali hospital, Mazandaran, Sari from November 2017 to September 2018. Iran. Inclusion criteria were birth weight of 2.5 to 4 grams, exclusive breastfeeding, and initiation of jaundice on the second day of life or thereafter, uncomplicated delivery, breastfeeding initiation by mother within the first 24 hours after birth, access to the parents for follow-ups, and oral tolerance of the neonate to deliver the medication (i.e., no nausea, vomiting or signs of obstruction the GI tract). Exclusion criteria were conjugated hyperbilirubinemia, hematocrit $>65 \%$ or hemoglobin $>21 \mathrm{~g} / \mathrm{dL}$, abnormal blood tests (reticulocyte count $>7 \%$, positive Coombs test, abnormal peripheral blood smear such as presence of spherocytes or elliptocytes), meconium staining, need for neonatal respiratory resuscitation at birth, obstetrical trauma, congenital abnormalities, intrauterine infections, severe jaundice in the first 24 hours of life, neonatal bleeding and/or cephalohematoma, ABO and/or Rh blood group incompatibility, self-medication of Cotoneaster manna by the mother/neonate, and diagnosis of any hemolytic condition in the neonate.

\section{Interventions}

The neonates in the intervention group were received Cotoneaster manna drop in the dosage of $1 \mathrm{~g} / \mathrm{d}$ divided into 3 doses every 8 hours for at least 3 days and the control group received placebo drops similar to Cotoneaster drop in appearance, color, consistency, and odor with the same dosage and timing intervals. The neonates received phototherapy in both groups. 


\section{Outcomes}

The primary outcome was TSB level which was measured at baseline and at 12 hours, 24 hours (day 1), and then daily for 9 days. The secondary outcomes were "discharge from hospital" evaluated from day three onward, "frequency of daily bowel movements", assessed from day 1 onward, "phototherapy duration", "admission duration", and side effects attributable to Cotoneaster including severe colic, diarrhea, and signs of dehydration. All the neonates were hospitalized for at least three days and a neonatologist to document primary and secondary outcomes performed daily visits. From day 4 , in case of $\mathrm{TSB}<10 \mathrm{mg} / \mathrm{dL}$, the neonates were discharged. In case of discharge from the hospital on day 4, the parents were asked to bring the neonate to the hospital on the next day (day 5) to check the neonate's TSB.

\section{Sample size}

To estimate the required sample size, considering the design of the study (i.e., the number of bilirubin measurements at baseline and thereafter), type I and II errors as 0.05 and 0.10 , and "clinical significance" or "minimal clinically important difference (MCID)" of $1 \mathrm{mg} / \mathrm{dL}$ for TSB (11) sample module by Stata (version 13.0) for ANOVA/ ANCOVA was used. This yielded a sample size of 40 patients in each study group. Considering a loss to followup rate of $25 \%$, the final sample size was determined as 50 newborns in each trial arm.

\section{Randomization and blinding}

Randomization was done by applying block balanced randomization method. A random allocation sequence was generated using www.randomization.com and block balanced randomization was used for random allocation of eligible neonates into two groups. To assure blindness, similarity of the interventions in the two groups was considered. Therefore, placebo was prepared in similar packages with all physical characteristics identical. The dosage and frequency of using the preparations were similar in both groups. The sole difference was related to the code (A or B) printed on the envelope and related medication package. Mothers of the neonates as well as all research team staff including receptionist, nurses, pediatrician who provided mothers with the necessary training and advice, as well as technicians and laboratory specialists who evaluated TSB levels were blinded to the two preparations and codes printed on the drops.

\section{Statistical analysis}

In order to compare baseline continuous variables between the intervention and control groups, the independent $t$ test (in case of normal distribution) and the Mann-Whitney $\mathrm{U}$ test (for the variables with non-normal distributions) were used. The chi-squared test was used to compare the baseline categorical variables between the two groups.
In the primary analysis, the primary outcome was compared at each measurement time point between the two groups by independent $t$ test with Bonferroni correction for multiple comparisons (12). The mean difference (MD) and the standardized mean difference (SMD) or Cohen's d were calculated for each paired comparison at different time points. The SMD values were categorized as follows; $<0.20=$ negligible effect size, 0.20 to $0.49=$ small effect size, 0.50 to $0.79=$ moderate effect size, and values $>0.80$ were regarded as large effect size (13). Additionally, in order to adjust the measured TSB levels after enrollment for baseline TSB level (i.e., baseline covariate), two one-way ANOVA (analysis of variance) models (full model and reduced model) were used. These two models differ in only one parameter (independent variable or potential confounder or covariate). In the full model, the TSB level measured (on day 3 ) was considered as the dependent variable, and the study groups were regarded as the independent variable, with baseline TSB level as the covariate. In the reduced model, baseline TSB level (covariate) was not included. A repeated-measures ANOVA was performed to detect changes of TSB levels within each study group. Of all 11 bilirubin measurements, only values obtained at baseline and hours 12, 24, 48, 72, and 120 (six measurements) were analyzed. As some neonates were discharged at other times and the related data were not available, other measurement times were not included. It should be added that "discharge from hospital" was a secondary outcome which was compared between the groups. Other measured times (hours 96, 144, 168, 192, and 216) were used for the analyses. In secondary analyses, discharge from hospital was compared between the groups using risk ratios with $95 \%$ confidence interval (CI). The analysis was performed based on per protocol (PP) and intention to treat (ITT) approaches to address the "loss to follow-up" effect.

\section{Results}

\section{Oral drop formulation and characterization}

Oral drop formulated and prepared with $1 \mathrm{~g} / \mathrm{mL}$ of the water-soluble content of manna, deionized water and preservative $(0.02 \%$ propyl paraben and $0.18 \%$ methyl paraben). Additionally, mannitol and total flavonoid contents in the oral drop have been calculated 53 and 1.6 $\mathrm{mg} / \mathrm{mL}$, respectively. The oral drop had a sweet taste and was clear with no colour.

\section{Clinical trial}

A total number of 100 eligible neonates with jaundice were included and randomized into the intervention (Cotoneaster drops) and the control (placebo drops) groups. Of those enrolled, only one neonate in the control group was withdrawn as per request of the parents for discharge on day 4 of the admission (Figure 1). In Table 1, maternal and neonatal characteristics in the intervention 
and control (comparison) groups are presented. As observed, no significant difference existed between the two groups regarding age, gender, weight, hemoglobin level, and frequency of pathologic jaundice. The neonatal age range in both groups was 2 to 8 days. The median neonatal age in the intervention and control groups were respectively 3.5 and 4 days. The highest frequency of TSB measurement was 11 times. Daily bowel movements and diaper weighting were recorded nine times. The neonates of both groups stayed at the hospital for at least 72 hours. Since the neonates were gradually discharged from day 4 onward, the analyses to determine the effect of the intervention on the primary outcome were performed until 72 hours after hospitalization.

\section{Primary outcome}

Table 2 presents TSB levels in neonates at 6 time points. It was observed no significant difference between intervention and placebo groups in TSB levels at the beginning as well as at 12 hours. However, from days one to five, mean TSB levels were significantly lower in the intervention group than in the control group with a mean difference ranging from 2.5 to $4 \mathrm{mg} / \mathrm{dL}$. After adjustment for baseline TSB (using the full and reduced ANOVA models), the adjusted differences did not change considerably owing to the small difference in baseline TSB between the two groups. Figure 2 shows the changes in mean TSB levels at seven time points (Baseline, 6 hours, 12 hours, and days 1 to 4 ). As seen, gradual declines in TSB levels post-intervention were recorded in both groups with more significant decline in the intervention group. The greatest differences of TSB levels between the groups were seen on days two and four. On day 4, 35 neonates in the intervention group and four in the control group were discharged.

\section{Secondary outcomes}

Table 3 presents secondary outcome of discharge from the hospital from day 4 . Most discharges occurred on day 4 as this rate was 11.5 times higher in the intervention group than in the control group. However, the degree of this differencelater (days 6 to 9 ) was not so great. Discharge rates were respectively $20 \%$ and $14 \%$ higher on days 6 and 7 in the intervention group compared to the control group with significant differences. On day 9, no significant difference was observed regarding hospital discharged either by PP or ITT approach. The median $\left(25^{\text {th }}, 75^{\text {th }}\right.$ percentiles $)$ hospitalization durations in the intervention and control groups were $3(3,4)$ and $4(4,5)$. This demonstrated a significantly lower duration with median of one day in the intervention group when compared to the control group $\left(P<0.0001, \chi^{2}=28.2\right)$. Median numbers of daily bowel movements in the study groups are depicted in Figure 2. AT baseline, the two groups were comparable (median $=$ 3 ) regarding this item. On days 1 to 4 , median daily bowel

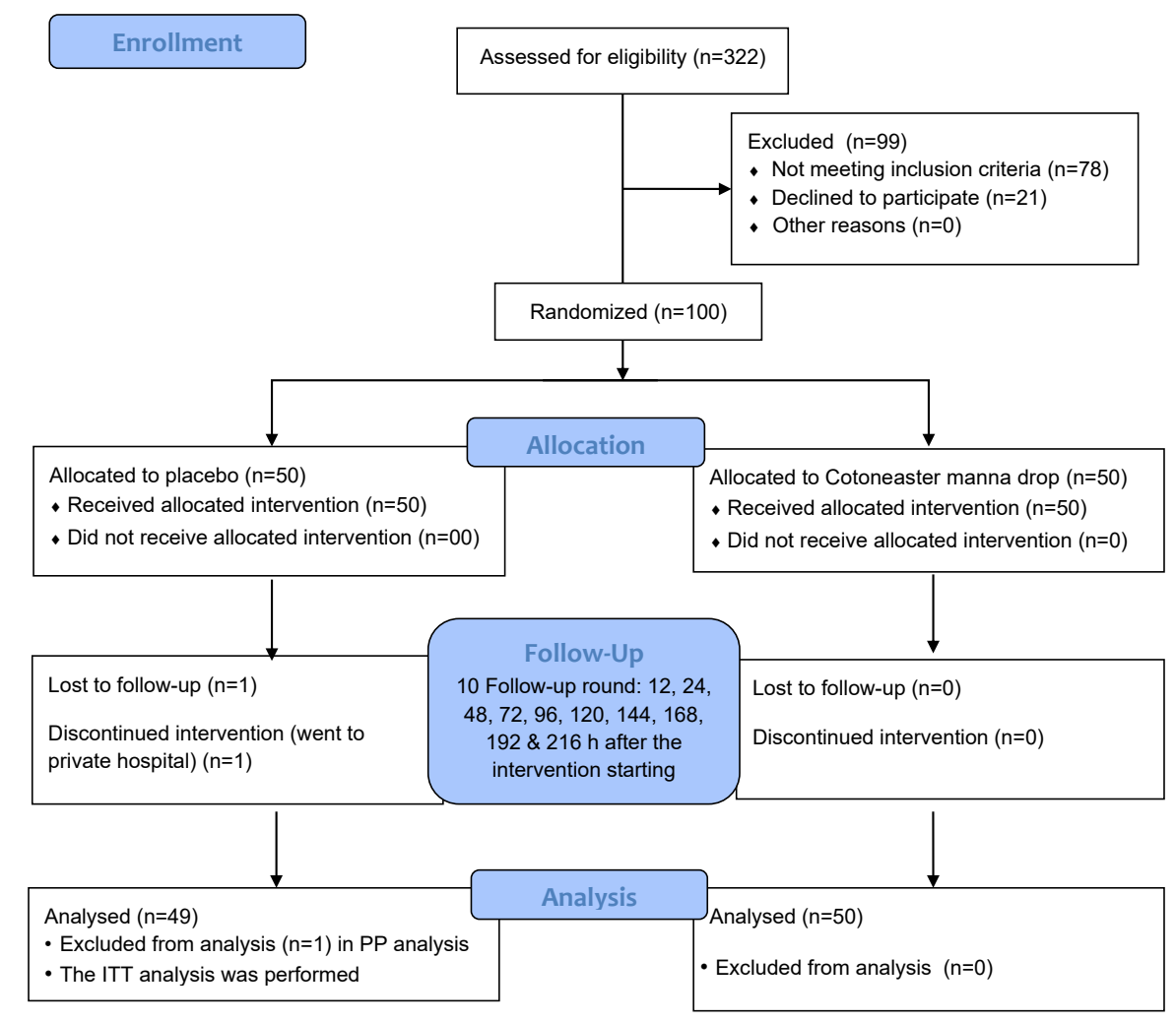

Figure 1. Flow diagram of the RCT from enrollment phase to outcomes measurement by two arms. This flowchart was prepared based on the primary outcome measurement. PP, per protocol; ITT, intention to treat. 
Table 1. Baseline maternal and neonatal characteristics by two trial arms

\begin{tabular}{|c|c|c|c|c|}
\hline Variable & Scale/categories & Intervention (Cotoneaster), $\mathrm{n}=50$ & Comparison (placebo), $\mathrm{n}=50$ & $P$ value \\
\hline \multicolumn{5}{|l|}{ Maternal } \\
\hline Mother's age (year) & Mean (SD) & $30.0(5.9)$ & $28.8(5.3)$ & $0.27^{\mathrm{a}}$ \\
\hline Delivery mode & Cesarean, No (\%) & $36(72.0)$ & $31(62.0)$ & $0.29^{b}$ \\
\hline Primipara & No $(\%)$ & $27(54.0)$ & $27(54.0)$ & $1.0^{\mathrm{b}}$ \\
\hline Gestational age (week) & Mean (SD) & $38.3(0.6)$ & $38.3(0.6)$ & $0.63^{\mathrm{a}}$ \\
\hline Oxytocin using & No. $(\%)$ & $3(6.0)$ & $3(6.0)$ & $1.0^{\mathrm{b}}$ \\
\hline Background disorder & No. $(\%)$ & $13(26.0)$ & $14(28.0)$ & $0.82^{\mathrm{b}}$ \\
\hline History of neonatal jaundice & No./No. of eligible (\%) & $8 / 23(34.8)$ & $11 / 23(47.8)$ & $0.37^{\mathrm{b}}$ \\
\hline History of phototherapy & No./No. of eligible (\%) & $4 / 23(17.4)$ & $5 / 23(21.7)$ & $0.71^{\mathrm{b}}$ \\
\hline History of blood exchange & No./No. of eligible (\%) & $1 / 23(4.4)$ & $1 / 23(4.4)$ & $0.76^{c}$ \\
\hline \multicolumn{5}{|l|}{ Neonatal } \\
\hline Gender & Male, No. (\%) & $28(56.0)$ & $27(54.0)$ & $0.84^{\mathrm{a}}$ \\
\hline Weight (g) & Mean (SD) & $3089.8(367.4)$ & $3146.4(326.2)$ & $0.42^{\mathrm{a}}$ \\
\hline Age (day) & Mean (SD) & $4.1(1.6)$ & $4.3(1.7)$ & $0.44^{\mathrm{a}}$ \\
\hline Hemoglobin (g/dL) & Mean (SD) & $15.9(1.7)$ & $15.8(1.6)$ & $0.79^{a}$ \\
\hline Main cause of hospitalization & Pathologic jaundice, No. (\%) & $26(52.0)$ & $23(46.0)$ & $0.55^{b}$ \\
\hline
\end{tabular}

${ }^{\text {a }}$ Calculated by independent $t$ test; ${ }^{\text {b }}$ Calculated by chi-square test; ${ }^{\text {c }}$ Calculated by Fisher's exact test.

Table 2. Total serum bilirubin (TSB) levels at six time points (baseline, 12 hours, $1^{\text {st }}, 2^{\text {nd }}, 3^{\text {rd }}$ and 5 th days following the interventions) by two trial arms

\begin{tabular}{|c|c|c|c|c|c|c|}
\hline Model & Time point & $\begin{array}{l}\text { Intervention (Cotoneaster) } \\
(n=50)\end{array}$ & $\begin{array}{c}\text { Comparison (placebo) } \\
(n=50)\end{array}$ & MD $(95 \% \mathrm{CI})$ & Cohen's d $(95 \%$ Cl $)$ & $P$ value $^{\mathrm{a}}$ \\
\hline \multirow{6}{*}{ Crude } & Baseline & $16.79(1.53)$ & $17.00(1.47)$ & $-0.20(-1.32,0.92)$ & $-0.14(-0.53,0.26)$ & 0.99 \\
\hline & $12 \mathrm{~h}$ & $15.78(1.41)$ & $16.20(1.51)$ & $-0.41(-1.54,0.71)$ & $-0.28(-0.67,0.12)$ & 0.96 \\
\hline & $24 \mathrm{~h}\left(1^{\text {st }}\right.$ day $)$ & $13.00(1.74)$ & $15.47(1.73)$ & $-2.47(-3.32,-1.63)$ & $-1.66(-2.12,-1.21)$ & $<0.001$ \\
\hline & $48 \mathrm{~h}$ ( $2^{\text {nd }}$ day $)$ & $9.96(1.58)$ & $13.09(1.66)$ & $-3.14(-4.26,-2.02)$ & $-1.94(-2.41,-1.46)$ & $<0.001$ \\
\hline & $72 \mathrm{~h}$ ( $3^{\text {rd }}$ day) & $7.73(1.44)$ & $11.20(0.89)$ & $-3.47(-3.94,-2.99)$ & $-2.33(-2.84,-1.82)$ & $<0.001$ \\
\hline & $120 \mathrm{~h}\left(5^{\text {th }}\right.$ day $)$ & $5.33(1.17)$ & $9.29(1.80)$ & $-3.96(-5.09,-2.83)$ & $-2.64(-3.18,-2.11)$ & $<0.001$ \\
\hline \multirow{6}{*}{ Adjusted $^{b}$} & Baseline & - & - & - & - & \\
\hline & $12 \mathrm{~h}$ & $15.86(0.89)$ & $16.12(0.89)$ & $-0.26(-0.61,0.10)$ & $-0.29(-0.68,0.10)$ & 0.85 \\
\hline & $24 \mathrm{~h}$ ( $1^{\text {st }}$ day $)$ & 13.07 (1.39) & $15.40(1.39)$ & $-2.33(-2.89,-0.1 .78)$ & $-1.68(-2.13,-1.22)$ & $<0.001$ \\
\hline & 48 h ( $2^{\text {nd }}$ day $)$ & $10.01(1.43)$ & $13.04(1.43)$ & $-3.03(-3.60,-2.46)$ & $-2.12(-2.60,-1.63)$ & $<0.001$ \\
\hline & 72 h ( $3^{\text {rd }}$ day) & $7.76(1.09)$ & $11.16(1.09)$ & $-3.40(-3.83,-2.97)$ & $-3.11(-3.69,-2.53)$ & $<0.001$ \\
\hline & $120 \mathrm{~h}\left(5^{\text {th }}\right.$ day $)$ & $5.36(1.43)$ & $9.26(1.45)$ & $-3.90(-4.47,-3.33)$ & $-2.71(-3.25,-2.16)$ & $<0.001$ \\
\hline
\end{tabular}

a Based on Bonferroni method; ${ }^{\text {b }}$ Adjusted for baseline TBR (calculated based on ANOVA/ANCOVA model).

movements were 4 in the intervention group and 3 in the control group with no significant difference. However, on day 5 , this was 4.5 in the intervention group and 2 in the control group $(P<0.05$; Table 4$)$. Mean $( \pm S D)$ of daily phototherapy duration in the intervention and control groups were respectively $19.4( \pm 0.9)$ and $18.5( \pm 0.9)$ hours $(P<0.0001 ; \mathrm{t}=4.99)$. The SMD of phototherapy duration was 1 hour ( $95 \% \mathrm{CI}=0.6$ to 1.4 hours) which demonstrates superiority of Cotoneaster compared to placebo. Mean $( \pm \mathrm{SD})$ total phototherapy hours (throughout the study) were respectively $61.6( \pm 11.1)$ and $92( \pm 25.3)$ hours in the intervention and control groups $(P<0.0001 ; \mathrm{t}=7.78)$. This demonstrated a difference of 30 hours between the two groups and the duration of phototherapy was shorter in the intervention group. The SMD of this variable between the two groups was 1.6 hours ( $95 \% \mathrm{CI}=1.1$ to 2 ) (Table 5$)$. No side effects of Cotoneaster or placebo were documented in any group. In terms of adherence to the treatments, both groups had a satisfactory adherence.

\section{Discussion}

Based on the findings of this parallel RCT, the difference in TSB levels in the intervention group compared to the control group became significant after a minimum of 24 hours from the onset of treatment. On day 1 , the difference in TSB level between the groups was at least $1 \mathrm{mg} / \mathrm{dL}$. Even after adjustment for baseline TSB levels, the difference in TSB on day 1 rose to more than $2 \mathrm{mg} / \mathrm{dL}$ and within the 95\% CI, the difference is higher than the MCID (minimal clinically important difference). In addition, comparison of the secondary outcome "discharge from hospital" showing TSB level $<10 \mathrm{mg} / \mathrm{dL}$ showed that of every two to eight neonates in the Cotoneaster group, one neonate met the criteria to be discharged from hospital. Because 


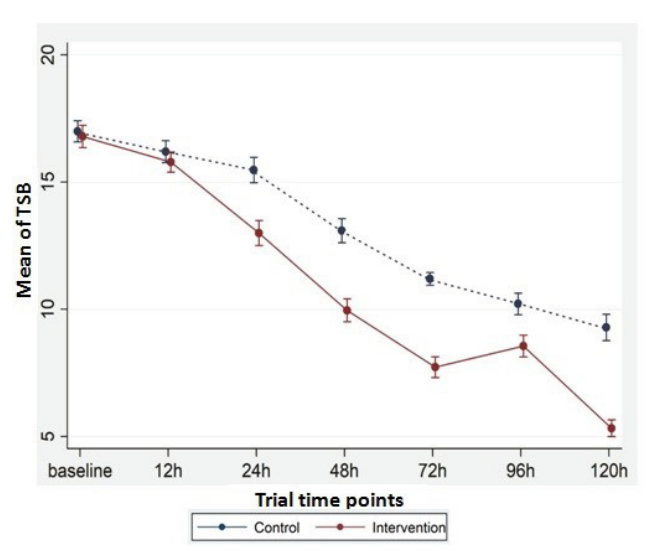

Figure 2. Trend of mean total serum bilirubin (TSB) levels (mg/dl) at different time-points by trial arms (the error bars indicated to $95 \%$ confidence interval of the means).

the number of cases who were lost to follow was minimal (only one newborn in the control group), it is unlikely that this phenomenon can have significant impact on the results. The analysis with ITT and PP approaches confirmed this and no difference was detected between these two approaches. Here, the Cotoneaster manna was only administered for the neonates. In a previous RCT to determine the efficacy of Cotoneaster manna (3 drops/ $\mathrm{kg}$ ), in addition to phototherapy in reducing TSB levels in neonates with jaundice (14), not only the neonates, but their mothers also received the Cotoneaster manna. The results of this study, compatible with our findings, showed that at 24 hours post-intervention, all treatment groups (mean TBR of $12.32 \mathrm{mg} / \mathrm{dL}$ in mother and neonate group, 12.23 $\mathrm{mg} / \mathrm{dL}$ in mother only group, and $11.01 \mathrm{mg} / \mathrm{dL}$ in neonate only group) had significantly lower TSB levels compared to control group with mean TSB of $13.82 \mathrm{mg} / \mathrm{dL}$. We did not detect superiority of Cotoneaster manna at 12 hours. This is in agreement with the above mentioned study (14), as the authors reported that at 12 hours, reduced TSB levels ( $13.95 \mathrm{mg} / \mathrm{dL}$ ) were only observed in the group where both mothers and neonates administered Cotoneaster manna compared to control group with a mean TSB of $14.88 \mathrm{mg} /$ dL. In another RCT to investigate the effect of Cotoneaster manna (5 drops/kg three times daily) on lowering TSB and indirect bilirubin levels in newborns with jaundice (15), comparable to our findings, the authors observed significant difference in TSB and indirect bilirubin at 24 hours post-intervention with no difference at 12 hours. However, in the presented results, we observed that the difference remained statistically significant on days 2 , 3 , and 5. Nevertheless in the mentioned study (15), no difference observed at 48 hours. Cotoneaster manna has laxative effect, owing to its important ingredient mannitol, and it is supposed that, this is by this mechanism that excretion of bilirubin is facilitated (16). However, the exact mechanism is not clear. As Cotoneaster manna has laxative effect, there is a theoretical concern that by increasing bowel movements in neonates, Cotoneaster manna induced dehydration. However, in our study, although diaper weights were higher in the intervention group, diarrhea or severe dehydration were not recorded in any neonate. This is in agreement with the previous trials that no major side effects occurred in the subjects $(14,15)$.

\section{Conclusion}

Cotoneaster manna drop at the dose of 1 gram per day when combined with phototherapy was a safe and superior method to placebo drops and phototherapy in

Table 3. Assessing trend of hospital discharge (as a secondary outcome) at five time points (4th, 6th, 7th, 8th and 9th days following the interventions) by two trial arms. All data are no (\%) of discharged neonates

\begin{tabular}{|c|c|c|c|c|}
\hline Time point & Intervention (Cotoneaster), $\mathbf{n = 5 0}$ & Comparison (placebo), $n=49$ & Risk Ratio $(95 \% \mathrm{Cl})^{a}$ & Risk Ratio $(95 \% \mathrm{Cl})^{\mathrm{b}}$ \\
\hline $4^{\text {th }}$ day $\left(96^{\text {th }}\right.$ hours $)$ & $35(70.0)$ & $3(6.1)$ & $11.43(3.76,34.74)$ & $11.67(3.84,35.47)$ \\
\hline $6^{\text {th }}$ day $\left(144^{\text {th }}\right.$ hours $)$ & $50(100.0)$ & $41(83.7)$ & $1.20(1.06,1.35)$ & $1.22(1.07,1.39)$ \\
\hline $7^{\text {th }}$ day $\left(168^{\text {th }}\right.$ hours $)$ & $50(100.0)$ & $43(87.8)$ & $1.14(1.03,1.27)$ & $1.16(1.04,1.30)$ \\
\hline $8^{\text {th }}$ day $\left(192^{\text {th }}\right.$ hours $)$ & $50(100.0)$ & 47 (95.9) & $1.04(0.98,1.11)$ & $1.06(1.00,1.14)$ \\
\hline $9^{\text {th }}$ day $\left(216^{\text {th }}\right.$ hours $)$ & $50(100.0)$ & $48(98.0)$ & $1.02(0.98,1.06)$ & $1.04(0.98,1.10)$ \\
\hline
\end{tabular}

a based on per protocol (PP) approach; ${ }^{\text {b }}$ based on intention to treat (ITT) approach.

Table 4. Median [Q1, Q3] of defecation numbers (per day) in 6 time points (baseline, 12 hours, $1^{\text {st }}, 2^{\text {nd }}, 3^{\text {rd }}$ and $5^{\text {th }}$ days following the interventions) by two trial arms

\begin{tabular}{|c|c|c|c|}
\hline Time point & Intervention (Cotoneaster), $\mathrm{n}=50$ & Comparison (placebo), $n=50$ & $P$ value $^{a}$ \\
\hline Baseline & $3[3,4]$ & $3[2,4]$ & 0.22 \\
\hline 24 h ( $1^{\text {st }}$ day) & $4[3,5]$ & $3[2,4]$ & 0.08 \\
\hline $48 \mathrm{~h}\left(2^{\text {nd }}\right.$ day $)$ & $4[3,5]$ & $3[2,4]$ & 0.08 \\
\hline 72 h ( $3^{\text {rd }}$ day) & $4[3,5]$ & $3[2,4]$ & 0.005 \\
\hline $96 \mathrm{~h}$ ( $4^{\text {th }}$ day $)$ & $4[4,5]$ & $3[2,4]$ & 0.03 \\
\hline $120 \mathrm{~h}$ ( $5^{\text {th }}$ day $)$ & $4.5[4,5]$ & $3[2,3]$ & 0.02 \\
\hline
\end{tabular}

a Based on median test (nonparametric test). 
Table 5. Daily or total phototherapy (PT) time distribution (hours) in the last follow-up by two trial arms

\begin{tabular}{|c|c|c|c|c|c|c|}
\hline Model & Time point & Intervention (Cotoneaster), $\mathrm{n}=50$ & Comparison (placebo), $n=50$ & MD $(95 \% \mathrm{CI})$ & Cohen's d $(95 \%$ Cl) & $P$ value $^{a}$ \\
\hline & Daily PT time & $18.5(0.9)$ & $19.4(0.9)$ & $0.9(0.5,1.2)$ & $1.0(0.6,1.4)$ & $<0.001$ \\
\hline \multirow[t]{2}{*}{ Crude } & Total PT time & $61.6(11.1)$ & $92.0(25.3)$ & $30.4(22.7,38.2)$ & $1.6(1.1,2.0)$ & $<0.001$ \\
\hline & Daily PT time & $18.6(0.5)$ & $19.3(0.5)$ & $0.8(0.6,1.0)$ & $1.4(1.0,1.8)$ & $<0.001$ \\
\hline Adjusted $^{b}$ & Total PT time & $61.7(17.9)$ & $91.9(17.9)$ & $30.2(23.1,37.4)$ & $1.7(1.2,2.1)$ & $<0.001$ \\
\hline
\end{tabular}

Note: The values are mean (SD).

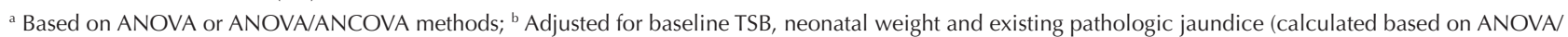
ANCOVA model).

management of neonatal hyperbilirubinemia. Significant difference in TSB level and lower levels in the intervention group was observed as soon as the second day of the study and remained throughout the study period. More neonates in the Cotoneaster manna group achieved TSB levels less than $10 \mathrm{mg} / \mathrm{dL}$ on day 4 and therefore significantly more neonates were discharged. Administration of Cotoneaster manna was not associated with major side effects such as dehydration or diarrhea that required cessation of its administration. We recommend administering Cotoneaster manna drop in neonatal jaundice as a safe method for management of hyperbilirubinemia.

\section{'What is already known?'}

C. discolor manna from Cotoneaster genus is presently used as an adjacent therapy (with phototherapy) for neonatal jaundice in Persian medicine in Iran.

\section{'What this study adds?'}

For the first time, C. nummularioides Pojark manna which has endemic with higher distribution growth in the Mazandaran province (our region) used as a new effective adjacent therapy (with phototherapy) for neonatal jaundice. Since C. nummularioides Pojark manna compared to Cotoneaster discolor, has more effective composition (mannitol); therefore may be used in neonatal jaundice with better treatment results.

\section{Limitations of the study}

The first limitation of the current study is that high risk neonates were not evaluated because we had to eliminate risk factors such as neonatal hemolytic disease in order to control the confounding factors. Second, the mechanism of the effect of the purgative manna was not evaluated. However, based on recent experiences, the effect of purgative manna can be due to the high percentage of sugars and especially alcoholic sugars based on mannitol in it and possibly increasing the frequency of neonate defecation in the intervention group indicates the laxative effect of purgative manna.

\section{Authors' contribution}

This work was carried out with the collaboration of all authors. MF and MA and RF were the principal investigators of the study. MF designed study, conducted clinical trial and data collection, did the assessment of documents and data analysis, wrote the first draft, did the reforms and managed the research. MA was supervisor of the research project, contributed with original data, critically edited and reviewed the manuscript. RF, second supervisor of the research project, cooperated in clinical process and assessment of neonates, cooperated in critically editing and reviewed the manuscript. SNM edited and reviewed the manuscript and performed data analysis. SSY and JA, and AD revised the manuscript and critically evaluated the intellectual contents. All authors have read and approved the content of the manuscript and confirmed the accuracy or integrity of any part of the work. All authors read and approved the final version of manuscript.

Conflicts of interest

There is no potential conflict of interest. The authors alone are responsible for the content and writing of the paper. The drug was provided to patients free of charge.

\section{Ethical issues}

The research was conducted in accordance with the tents of the Declaration of Helsinki. The Ethics Committee of Mazandaran University of Medical Sciences approved this study. The institutional ethical committee at Mazandaran University of Medical Sciences accepted all study protocols (\#IR. MAZUMS.REC.1394.1316). Accordingly, written informed consent was taken from all parents of participants before any intervention. This study was a part of Ph.D thesis of Moloud Fakhri at this university. The trial protocol was also approved by the Iranian Registry of Clinical Trials (identifier: IRCT20160531028188N4; https://en.irct.ir/trial/27765). Moreover, ethical issues (including plagiarism, data fabrication, double publication) have been completely observed by the authors.

Funding/Support

This study was funded by Mazandaran University of Medical Sciences in Sari, Iran (\#94-1-19-1316).

\section{References}

1. Mitra S, Rennie J. Neonatal jaundice: aetiology, diagnosis and treatment. Br J Hosp Med (Lond). 2017;78:699-704. doi: 10.12968/hmed.2017.78.12.699.

2. Porter ML, Dennis BL. Hyperbilirubinemia in the term newborn. Am Fam Physician. 2002;65:599-606.

3. Bhutani VK, Johnson LH, Keren R. Diagnosis and management of hyperbilirubinemia in the term neonate: for a safer first week. Pediatr Clin North Am. 2004;51:843-61. doi: 10.1016/j. pcl.2004.03.011.

4. Sgro M, Campbell DM, Kandasamy S, Shah V. Incidence of chronic bilirubin encephalopathy in Canada, 2007-2008. Pediatrics. 2012;130:e886-90. doi: 10.1542/peds.2012-0253.

5. Chen Z, Zhang L, Zeng L, Yang X, Jiang L, Gui G, et al. Probiotics supplementation therapy for pathological neonatal jaundice: a systematic review and meta-analysis. Front Pharmacol. 2017;8:432. doi: 10.3389/fphar.2017.00432.

6. Zeng J, Wang SJ, Li YM, Li HS, Luo Q, Huang YY, et al. Yinzhihuang oral liquid in the treatment of neonatal 
jaundice: a meta-analysis. Pharm Biol. 2017;55:554-9. doi: 10.1080/13880209.2016.1262432.

7. Kicel A, Michel P, Owczarek A, Marchelak A, Zyzelewicz D, Budryn G, et al. Phenolic profile and antioxidant potential of leaves from selected Cotoneaster Medik. Species. Molecules. 2016;21(6). doi: 10.3390/molecules21060688.

8. Holzer VM, Lower-Nedza AD, Nandintsetseg M, Batkhuu J, Brantner AH. Antioxidant constituents of Cotoneaster melanocarpus Lodd. Antioxidants (Basel, Switzerland). 2013;2(4):265-72. doi: 10.3390/antiox2040265.

9. Hercberg S, Galan P. Nutritional anaemias. Baillière's Clinical Haematology. 1992;5:143-68. doi: 10.1016/S09503536(11)80039-9.

10. Fakhri M, Farhadi R, Mousavinasab N, Hosseinimehr SJ, Yousefi S, Davoodi A, et al. Preventive effect of purgative manna on neonatal jaundice: a double blind randomized controlled clinical trial. J Ethnopharmacol. 2019;236:240-9. doi: 10.1016/j.jep.2019.03.009.
11. Makay B, Duman N, Ozer E, Kumral A, Yeșilirmak D, Ozkan $H$. Randomized, controlled trial of early intravenous nutrition for prevention of neonatal jaundice in term and near-term neonates. J Pediatr Gastroenterol Nutr. 2007;44:354-8. doi: 10.1097/mpg.0b013e31802b31f2.

12. McDonald JH. Handbook of Biological Statistics. Baltimore: Sparky House Publishing; 2009.

13. Cohen J. Statistical power analysis for the behavioural sciences. Hillsdale, NJ: Erlbaum; 1988.

14. Rafieian-Kopaei M, Khoshdel A, Kheiri S, Shemian R. Cotoneaster: A Safe and Easy Way to Reduce Neonatal Jaundice. J Diag Res Stud 2016; 10: 12-15.

15. Ameli Z, Assarroudi A, Akrami R. Effect of bilineaster drop on neonatal hyperbilirubinemia. Care Based Stud. 2017;6:46-70.

16. Kicel A. An overview of the genus Cotoneaster (Rosaceae): phytochemistry, biological activity, and toxicology. Antioxidants (Basel). 2020;9:1002. doi: 10.3390/antiox9101002. 